\title{
Detection and Typing of Human Papillomaviruses Combining Different Methods: Polymerase Chain Reaction, Restriction Fragment Length Polymorphism, Line Probe Assay and Sequencing
}

\author{
Nina MILUTIN GAŠPEROV, Ivan SABOL, Mihaela MATOVINA, Šime SPAVENTI, Magdalena GRCE* \\ Department of Molecular Medicine, Rudjer Boskovic Institute, Zagreb, Croatia
}

Running title: Human Papillomaviruses Identification by Different Methods

\author{
*Corresponding author: \\ E-mail: grce@irb.hr \\ Phone: +38514561110 \\ Fax: +38514561010
}

Key words: Human papillomavirus, consensus polymerase chain reaction, restriction fragment length polymorphism, line probe assay, sequencing, type-specific polymerase chain reaction

Abbreviations: High-grade Squamous Intraepithelial Lesion (HSIL), High-Risk (HR), Human papillomavirus (HPV), Line Probe Assay (LiPA, Innogenetics), Polymerase Chain Reaction (PCR), Restriction Fragment Length Polymorphism (RFLP)

\section{Summary}

The identification of the etiological factor of many cervical precancerous lesions and cervical cancer, the Human papillomavirus (HPV) is widely used. In this study, we evaluated the consensus and type-specific (TS) Polymerase Chain Reaction (PCR), Restriction Fragment Length Polymorphism (RFLP), Line Probe Assay (LiPA, Innogenetics) and sequencing to determine the HPV types in cervical specimens. Out of 690 High-grade Squamous Intraepithelial Lesion (HSIL) samples, $86.7 \%$ were HPV positive and 13.3\% HPV negative by consensus primers (MY09/MY11, L1C1/L1C2-1/L1C2-2 and/or GP5/6) directed PCR. Out of 598 HPV positive samples, 85.3\% were typed by TS-PCR being HPV 6/11, 16, 18, 31 and/or 33, while $14.7 \%$ remained untyped. The most prevalent HPV type in the study group was HPV 16, identified in $35.5 \%$ cases, while HPV 31 was the second most frequent HPV type with a prevalence of $10.5 \%$. They were followed by HPV types 6/11, 33 and 18 with a prevalence of $7.4 \%, 6.2 \%$ and $4.9 \%$, respectively. Multiple HPV infections with two or more HPV types $(6 / 11,16,18,31$ and/or 33$)$ were found in $9.4 \%$ cases. A subset of 88 samples was further typed by RFLP and LiPA to determine the rare HPV types in HSIL samples. The most frequent low abundant HPV types in single infections in decreasing order were HPV 53, 58, 66, 56 and 52, while HPV 51 was the most frequent low abundant HPV type found in multiple HPV infections. Multiple HPV infections were mostly found by LiPA in $27.3 \%$ cases versus $14.8 \%$ cases found by RFLP. The perfect agreement between RFLP and LiPA assay pair was observed only for HPV types 16, 18, 34 and 59 (Kappa value of 1). For other HPV types, the inter-assay agreement ranged from very good to no agreement indicating that neither assay is perfect. Sequencing was performed for 33 samples in cases where both RFLP and LiPA were inconclusive. Sequencing was shown to be a very good method in case of single HPV infection but not applicable in case of multiple HPV infections. Both RFLP and LiPA are good assays for epidemiological studies, although RFLP being cumbersome and time-consuming is less applicable than LiPA. Careful consideration has to be made before the implementation of either HPV typing methods in clinical laboratories. 


\section{Introduction}

Human papillomaviruses (HPV) have been recognized as an etiologic factor of a variety of widespread human cancers, anogenital and head and neck cancers. ${ }^{1-3}$ Until now, more than 100 HPV types have been identified and fully sequenced. ${ }^{4}$ Approximately $40 \mathrm{HPV}$ types infect the anogenital tract and a few are found in anogenital cancer biopsy specimens, notably cervical cancer. Oncogenic HPVs have been identified in almost all cervical cancer biopsies, i.e. $99.7 \%$ cases, ${ }^{5}$ what makes them undoubtedly the cause of the disease. According to the last epidemiological classification, 15 HPV types: 16, 18, 31, 33, 35, 39, 45, $51,52,56,58,59,68,73$ and 82 are considered as highly oncogenic (high-risk [HR]), and HPV types 26, 53 and 66 as probably oncogenic, while HPV types $6,11,40,42,43,44,54,61,70,72,81$ and CP6108 are classified as viruses with low oncogenic potential (low-risk [LR]). ${ }^{6} \mathrm{HPV} 16$ and 18 alone are found in nearly $60 \%$ and $17 \%$ of cervical cancer cases worldwide, respectively, while all other HPV types are found in less than $1-7 \%$ cases each. ${ }^{7}$ In addition, multiple HPV infections seem to be very common and in previous studies were found in almost $7 \%$ of women with precancerous cervical lesions. ${ }^{8,9}$

Currently, DNA amplification mediated by the polymerase chain reaction (PCR) is the most specific and the most sensitive for revealing the presence of otherwise undetectable quantities of HPV DNA. Several methods for HPV typing by PCR were developed in research laboratories. The identification of HPV types may be accomplished either by Type-Specific (TS) based PCR (TS-PCR) or by analysis of consensus PCR products through hybridisation with TS probes fixed on solid support (i.e. nylon or nitrocellulose strips or chip), Restriction Fragment Length Polymorphism (RFLP) or sequencing. ${ }^{10}$ The TS-PCR ${ }^{8,11,12}$ is a useful tool for identification of individual HPV types, especially HR types, and it is a good approach if only a few types need to be identified. As at least 15 HR HPV types are important for epidemiological studies TS-PCR is not the best approach, because it is time consuming and not at all cost-effective for the determination of so many HPV types. It is why several group of researchers developed the line blot assays based on the hybridisation with specific probes fixed on strip and consensus HPV amplicons generated with different biotin-labelled primers: MY09/11/HMB01, ${ }^{13} \mathrm{SPF} 1 / 2,{ }^{14}$ and GP5 $+/ 6+,{ }^{15}$ allowing the detection of as much HPV types as specific probes applied on the strip. The line blot assays showed to be more appropriate for large scale HPV typing.

The RFLP method enables HPV typing by the analysis of difference in DNA fragments length after cleavage of the consensus HPV amplicons with specific restriction endonucleases, while sequencing enables determination of all nucleotides alignment of the full PCR product length. Both methods are very informative but time consuming and inappropriate in case of multiple infections. Generally, they are used for research purpose rather than for routine clinical testing.

In this study, we evaluated the consensus and TS-PCR, RFLP, sequencing and commercially available Line Probe HPV typing Assay (LiPA, Innogenetics) to determine the HPV types in cervical specimens.

\section{Material and Methods}

Study group

We analysed 690 DNA from cervical scrapes of women with cytological diagnosis of High-grade Squamous Intraepithelial Lesions (HSIL) ${ }^{16}$ collected in different gynaecological clinics in Zagreb (Croatia). Cervical scrapes were collected with cyto-brushes, placed in 3-5 $\mathrm{ml}$ of phosphate-buffered saline $(\mathrm{pH} 7.2)$ and frozen at $-20^{\circ} \mathrm{C}$ until analysis.

\section{DNA preparation}

DNA from cervical cell samples was isolated as described previously. ${ }^{17}$ Briefly, cell suspensions were centrifuged $(3,000 \mathrm{~g}, 10 \mathrm{~min})$ and resuspended in lysis buffer $(10 \mathrm{mM}$ Tris- $\mathrm{HCl} ; \mathrm{pH} 7.5,1 \mathrm{mM}$ EDTA, $\mathrm{pH} 7.9 ; 0.5 \% \mathrm{SDS})$. Proteinase $\mathrm{K}(100 \mu \mathrm{g} / \mathrm{ml})$ was added on pelleted cells and it was incubated overnight at $37^{\circ} \mathrm{C}$ or $2 \mathrm{~h}$ at $56^{\circ} \mathrm{C}$. Standard $\mathrm{NaCl}$ or phenol-chloroform extraction and ethanol precipitation were used for DNA purification. The DNA precipitate was resuspended in 50-100 $\mu \mathrm{L}$ of tridistillated sterile water. DNA concentration and the quality were determined both spectrophotometrically and by electrophoresis on $1 \%$ agarose gels. ${ }^{18}$ 
The amplification reactions included three sets of consensus primers: MY09/MY11, L1C1/L1C21/L1C2-2 primers in a 2:1:1 ratio and GP5/6 consensus primers. TS primers for HPV 6/11, 16, 18, 31 and 33 were also used in two separate multiplex PCR reactions, i.e. HPV 6/11 with 31 and HPV 16 and 18 with 33. ${ }^{8,19,20}$ To control the quality of the isolated DNA and the absence of PCR inhibitors, we amplified the 268 bp sequence of $\beta$-globin gene using PC04/GH20 primers ${ }^{21}$ in a multiplex PCR with MY primers. Each amplification reaction was carried out in a total volume of $20 \mu \mathrm{L}$. The reaction mixture contained tridistillated sterile water, $10 \mathrm{mM}$ Tris- $\mathrm{HCl}(\mathrm{pH} 8.3), 50 \mathrm{mM} \mathrm{KCl}, 1.5 \mathrm{mM} \mathrm{MgCl} 2,100 \mu \mathrm{M}$ of each dNTP, $0.15 \mu \mathrm{M}$ of each TS primer, $0.12 \mathrm{U}$ AmpliTaq Gold DNA Polymerase (Roche) and $100 \mathrm{ng}$ of each DNA. Each PCR was carried out with first denaturation step at $95^{\circ} \mathrm{C}$ for $10 \mathrm{~min}$ and final extension at $72^{\circ} \mathrm{C}$ for 15 $\mathrm{min}$. The conditions and the number of denaturation-annealing-extension cycles were different with each set of primers. ${ }^{8}$

Aliquots of each PCR product $(10 \mu 1)$ were analysed by electrophoresis on $2 \%$ agarose gels stained with ethidium bromide. The amplified products were identified by UV irradiation of the gels and photographed by Image Master VDS (Pharmacia Biotech).

\section{Restriction Fragment Length Polymorphism (RFLP)}

A subset of samples, which remained untyped by TS-PCR were further analysed by restriction fragment length polymorphism (RFLP) as described previously. ${ }^{22-24}$ Briefly, an aliquot (4-12 $\mu$ l) of an MY09/MY11 PCR product was used for each digestion. The restriction enzymes used for these analyses were DdeI, DraI, PstI, Sau3AI, BamHI, HaeIII and/or RsaI (Roche). RFLP can discriminate between 43 HPV types and 2 subtypes (HPV 6b, 11, 13, 16, 18, 26, 30-35, 39, 40, 42-45, 51-59, 61, 62, 64, 66-73, 8184 and 89). ${ }^{23}$ Aliquots of PCR products were incubated at $37^{\circ} \mathrm{C}$ overnight with addition of 2-10 U of restriction enzyme and corresponding buffer ${ }^{23}$ and overlaid with mineral oil. Reactions were carried out in a total volume of $25 \mu \mathrm{l}$. Restriction fragments were analysed on $8 \%$ polyacrylamide gel during $3 \mathrm{~h}$ of electrophoresis at $500 \mathrm{~V}$ and stained with silver. ${ }^{18}$ They were also analysed on Spreadex EL 800 (Elchrom Scientific) gel during $150 \mathrm{~min}$ of electrophoresis at $120 \mathrm{~V}$ and $55^{\circ} \mathrm{C}$ and stained with SYBR Gold, according to manufacturer's protocol (Molecular Probes, Eugene). The gels were photographed and analysed on Image Master VDS.

\section{Line Probe Assay (LiPA)}

The same subset of samples, which was analysed by RFLP was also tested with the commercially available HPV typing assay LiPA (Innogenetics). ${ }^{25}$ In this assay, 28 oligonucleotide probes that recognize 26 different HPV types $(6,11,16,18,31,33-35,39,40,42-45,51-54,56,58,59,66,68,73,70$ and 74) were enzymatically tailed with poly (dT) and applied as horizontal lines to the nitrocellulose membrane strips. The PC04/GH20 primers for $\beta$-globin gene were used for internal control, while SPF 10 primers were used for HPV DNA amplification. The PCR conditions were those recommended by manufacturer (Innogenetics). Amplified biotinylated SPF 10 PCR products were denatured under alkaline conditions and added to the strip in an appropriate hybridization buffer. After hybridization and stringent washing, the hybrids were detected by addition of a streptavidin-conjugate and a substrate, generating a purple precipitate at the probe line. The strip results were interpreted by comparing the hybridization pattern to the provided template.

\section{Sequencing}

A subset of HPV positive samples, which showed ambiguous result with either RFLP or LiPA, were amplified with MY09/MY11 primers and purified with QIAquick PCR Purification Kit (Qiagen) according to the manufacturer's instructions. After purification, $2 \mu \mathrm{l}$ of each amplicon was tested by electrophoresis on $2 \%$ agarose gels for the evaluation of the quality and the quantity of amplified DNA. The purified amplicons were sequenced at the local core sequencing facility (ABI PRISM 310 Genetic Analyzer, Applied Biosystems) of Rudjer Boskovic Institute - Zagreb using the BigDye Terminator v1.1 Cycle Sequencing Kit (Applied Biosystems) and forward PCR primer. The resulting sequences were compared with HPV sequences of known types using the basic local alignment search tool from the NCBI website (www.ncbi.nlm.nih.gov/blast/Blast.cgi). ${ }^{26}$

\section{Statistical analysis}

The RFLP and LiPA assays were compared using unweighted Kappa statistic (MedCalc version 7.3.0.1, MedCalc Software, Mariakerke, Belgium). A Kappa (K) value of 0 indicates no agreement better than chance and a value of 1 indicates perfect agreement. $K$ values from 0 to $0.20,0.21$ to $0.40,0.41$ to 0.60 , 0.61 to 0.80 and 0.81 to 1.00 indicate poor, fair, moderate, good and very good strength of agreement, respectively. 


\section{Results}

Detection and typing of common HPV types by consensus and TS-PCR

Each DNA specimen from cervical cell samples was positive for the $\beta$-globin gene and as such suitable for further HPV DNA detection and typing by different methods.

Out of 690 HSIL samples, $598(86.7 \%)$ were HPV positive and 92 samples $(13.3 \%)$ gave the negative result in PCR with consensus primers (MY09/MY11, L1C1/L1C2-1/L1C2-2 and/or GP5/6) and TS primers for HPV 6/11, 16, 18, 31 and 33 (Fig. 1). Out of HPV positive samples, 85.3\% (510/598) were typed by TS-PCR being HPV 6/11, 16, 18, 31 and/or 33, while $14.7 \%(88 / 598)$ were not determined. The most prevalent HPV type in the study group was HPV 16, identified in $35.5 \%$ cases (245/690), while HPV 31 was the second most frequent HPV type with a prevalence of $10.5 \%(72 / 690)$. They were followed by HPV types 6/11, 33 and 18 with a prevalence of $7.4 \%(51 / 690), 6.2 \%(43 / 690)$ and $4.9 \%(34 / 690)$, respectively. Multiple HPV infections with two or more HPV types $(6 / 11,16,18,31$ and/or 33) were found in $9.4 \%(65 / 690)$ cases.

\section{Typing of rare HPV types by RFLP, LiPA and sequencing}

A subset of samples ( 88 out of 690) that were positive with consensus PCR but negative with TSPCR for HPV 6/11, 16, 18, 31 and 33 were further analysed with RFLP and LiPA, while 32 of them, for which we could not determine the exact type with those methods, were additionally analysed by sequencing (Table 1). The combined results of HPV typing by RFLP, LiPA and sequencing are presented in Fig. 2. The most frequent low abundant HPV types in single infections were HPV 53, 58, 66, 56 and 52 found in 13.6\% (12/88), 10.2\% (9/88), 8\% (7/88), 6.8\% (6/88) and 4.5\% (4/88) samples, respectively. Multiple HPV infections with two or more low abundant HPV types were found in $20.5 \%$ (18/88) cases. The most prevalent types in multiple infections were HPV 66, 51, 52 and 53 in $6.8 \%$ (6/88), 5.7\% (5/88), 4.5\% (4/88) and $4.5 \%$ (4/88) cases, respectively. Although rather frequently found in multiple infections, HPV 51 was not detected among single HPV infections. HPV type was not precisely determined in 3 specimens (3.4\%). In two of those specimens sequencing detected a rare HPV isolate IS223, while RFLP and LiPA determined it as HPV 73. In one specimen sequencing detected a rare HPV isolate SDL105, while LiPA determined it as HPV 70 and RFLP failed to determine it (Table 1).

\section{Comparison of HPV typing by RFLP and LiPA}

Out of 88 samples analysed by RFLP and LiPA, single HPV infections were detected in $59(67 \%)$ and $57(64.8 \%)$ cases by RFLP method and LiPA, respectively (Table 2). Multiple HPV infections were determined in $14.8 \%$ (13/88) by RFLP, and in $27.3 \%$ (24/88) by LiPA. In $18.2 \%$ (16/88) of cases RFLP method could not determinate any specific HPV type, while LiPA was not successful in typing $8 \%(7 / 88)$ cases.

Inter-assay agreement (Kappa [K]) was calculated with a 95\% confidence interval (CI) for RFLP and LiPA assay pair for all HPV types detected by these two methods (Table 3). The perfect agreement between RFLP and LiPA assay pair was observed for HPV types 16, 18, 34 and 59 (K value of 1). For HPV types 53,56 and 58 a very good strength of agreement (K ranging from 0.805 to 0.838 ) was observed. The inter-assay agreement for HPV 31 and 33 was good, with K values of 0.794 and 0.656 , respectively. HPV types 66 and 73 had moderate inter-assay agreement (K values of 0.453 and 0.542 , respectively). A fair strength of inter-assay agreement was calculated for HPV types 39, 45, 52 and 68 (K ranging from 0.272 to 0.389). For HPV types $26,35,40,42,51,55,61,62,64,67,70,83$ and 84 , no agreement better than chance was observed ( $\mathrm{K}$ value of 0 ).

When we compared RFLP and LiPA according to the type of HPV and the number of identified HPV types, both in single and multiple infections, we found complete agreement between these two methods in $38.6 \%$, partial agreement in $20.5 \%$ and disagreement in $37.5 \%$ cases. For 3 samples $(3.4 \%)$, it was not possible to compare these two methods (Table 1, Fig. 3).

The main causes of disagreement between RFLP and LiPA findings are the following (a) RFLP could not determine HPV types determined by LiPA in 41.7\% (15/36) cases, (b) both assays identified different HPV type in $19.4 \%$ (7/36) cases because the type detected by RFLP cannot be detected with LiPA and probably because RFLP is not sensitive enough to detect the other type detected by LiPA, and (c) RFLP could not resolve multiple HPV infections determined by LiPA in $16.7 \%(6 / 36)$ cases. The minor causes of disagreement between RFLP and LiPA consist of (a) RFLP detecting HPV types that LiPA a priori cannot detect $(5.6 \%$; 2/36), (b) HPV types determined by RFLP but not by LiPA even though it should (5.6\%; 2/36), (c) unresolved multiple HPV infections by RFLP and undetermined by LiPA $(5.6 \%$; 2/36), (d) different HPV type detected by these two methods $(2.8 \%$; 1/36), and (e) HPV type undetermined by both methods $(2.8 \% ; 1 / 36)$. 


\section{Comparison of sequencing with RFLP and LiPA results}

Out of 32 samples that were analysed by sequencing, 3 samples $(9.4 \%)$ showed complete agreement in detecting single HPV type with both, RFLP and LiPA results, while partial agreement with both methods was found in 1 sample (3.1\%) (Table 1). Complete agreement between RFLP and sequencing results was found in 14 samples (43.8\%), while complete agreement between LiPA and sequencing was found in 5 cases $(15.6 \%)$. Four samples showed complete disagreement of sequencing results in comparison with both, RFLP and LiPA (12.5\%), one (3.1\%) showed partial agreement with LiPA results, 3 of them $(9.4 \%)$ were detected by sequencing as rare HPV isolates that could not be resolved by RFLP and/or LiPA and 1 sample (3.1\%) was not determined by sequencing.

\section{Discussion}

This study was designed to detect and determine the most common and rare HPV types in HSIL samples by combining different methods: consensus and TS-PCR, RFLP, sequencing and a commercially available line probe HPV typing assay (LiPA, Innogenetics). The purpose of this study was also to evaluate the value of above listed different HPV typing methods and to identify the less common HPV types that might be of clinical significance in precancerous cervical lesions.

Herein, 690 cervical specimens collected from Croatian women with HSIL diagnosis were analysed. Detection of HPV by consensus PCR found $86.7 \%$ HPV positive and 13.3\% HPV negative samples. The high percentage of HPV positive HSIL samples was in line with the worldwide meta-analysis. ${ }^{27,28}$ Typing of the most common HPV types (HPV 6/11, 16, 18, 31 and 33) by TS-PCR identified HPV 16, as the most prevalent type among Croatian women, and that was also found worldwide. The second most commonly found HR HPV type among Croatian women was HPV 31, which was also found on the second place in overall world HSIL cases. The following HPV types in this study in decreasing order were HPV 6/11, 33 and 18, while in the worldwide meta-analysis those were HPV 58, 18 and 33; HPV 58 being very common type in Asia and South and Central America. The LR HPV 6/11 was frequently found in Croatian cervical HSIL samples, as well as HPV 6 was found in North America as a second most common HPV type among HSIL samples. ${ }^{28}$

The multiple HPV infection, found in $9.4 \%$ cases by TS-PCR for HPV 6/11, 16, 18, 31 and 33, only, is probably underestimated because approximately $40 \mathrm{HPV}$ types infect the anogenital tract. Indeed, multiple HPV infection in our study subset of 88 HSIL samples positive by consensus PCR and negative by TS-PCR for $6 / 11,16,18,31$ and 33 was found in higher percentage of $20.5 \%$ by combining methods: RFLP, LiPA and sequencing. This high percentage of multiple infections in HSIL was reported in previous studies. ${ }^{29-32}$

Among the samples analysed by RFLP, LiPA and sequencing, the most frequent low abundant HPV types in single infections in decreasing order were HPV 53, 58, 66, 56 and 52. Similar frequencies of low abundant HPV types in HSIL samples were reported in previous studies. ${ }^{32}$ In case of multiple HPV infections with two or more low abundant HPV types, we found a slightly different situation. The most prevalent types in multiple infections were HPV 66, 51, 52 and 53. Interestingly, rather frequently found in multiple infections, mostly identified by LiPA, HPV 51 was rarely detected as single HPV infection, also exclusively by LiPA method. Moreover, in two samples where LiPA found single infection with HPV 51, RFLP and sequencing, both, identified other HPV types, i.e. HPV 61 in one case and HPV 62 in other (Table 1). The question could be raised: are these positive findings by LiPA really HPV 51 or they are results of possible cross-hybridization with other HPV types present in multiple infections. However, HPV 51 was also frequently found in other studies in which other line blot assays for HPV typing ${ }^{29-32}$ were used, which indicate that it can be considered as common HPV type, especially in HSIL specimens.

Combining RFLP, LiPA and sequencing, 3 specimens remained with uncertain HPV type. In two of those specimens sequencing detected a rare HPV isolate IS223, while RFLP and LiPA determined it as HPV 73. Perhaps the IS223 isolate is very close to HPV type 73. In another of those specimens sequencing detected a rare HPV isolate SDL105, while LiPA determined it as HPV 70 and RFLP failed to determine it as any HPV type. This finding is also suggesting that the SDL105 isolate is very close to HPV type $70 .{ }^{4}$

When we compared only RFLP and LiPA, we found that while both methods showed rather similar frequencies in determination of single HPV infections (67\% versus $64.8 \%$, respectively), LiPA detected multiple infections almost twice more often than RFLP (27.3\% versus $14.8 \%$, respectively). The fact is that RFLP is not a method of choice for detection of multiple HPV infections, contrary to LiPA. 
Inter-assay agreement (Table 3) for RFLP and LiPA assay pair for each HPV type detected by these two methods indicate perfect agreement for HPV types 16, 18, 34 and 59 (K value of 1), signifying that these types were almost equally detected by both methods. This finding is encouraging since HPV 16, 18 and 59 belong to HR HPV types of which HPV 16 and 18 are the most frequently found types in HSIL samples and in cervical cancer worldwide. ${ }^{27,28}$ For HPV types 53,56 and 58 a very good strength of agreement (K ranging from 0.805 to 0.838 ) was observed, which is also very good result since HPV 56 and 58 are HR and HPV 53 probably HR. The inter-assay agreement for HR HPV 31 and 33 was good, with K values of 0.794 and 0.656 , respectively. The subset of samples subjected to inter-assay analysis was a priori depleted of HPV 31 and 33, so the $\mathrm{K}$ values in these cases are not representative as it is referring to a low number of positive samples. HPV types 66 and 73 had moderate inter-assay agreement (K values of 0.453 and 0.542 , respectively), although each of them can be identified by both methods. Herein, either RFLP underestimates the presence of HPV 66 and 73 or LiPA overestimated it. Unfortunately, a fair strength of inter-assay agreement was calculated for HPV types $39,45,52$ and 68 (K ranging from 0.272 to 0.389), which are relatively frequently found in this study group (Fig. 2). In this case also, either RFLP underestimates the presence of these types or LiPA overestimated it. For HPV types 26, 35, 40, 42, 51, 55, 61, 62, 64, 67, 70, 83 and 84 no agreement better than chance ( $\mathrm{K}$ value of 0 ) was observed, probably because of the low number of cases positive for each of these types, except for HPV 51, which was found in a high proportion in multiple HPV infections by LiPA. Some of the observed discrepancies can be attributed to different sensitivity of consensus primers used, i.e. generating amplicons of different size; from our previous observational study comparing different consensus HPV primers shorter PCR products were more efficiently amplified..$^{20}$ Because of these important discrepancies between RFLP and LiPA (Fig. 3) for the detection of, especially, HPV types 39, 45, 51, 52, 66, 68 and 73, the evaluation of the findings by RFLP and LiPA, with a third typing method would be appropriate. Neither of those HPV typing assays is perfect, mainly because RFLP could not identify HPV types determined by LiPA (41.7\% cases), RFLP identified different HPV types, which are untypable by LiPA (19.4\% cases), and RFLP could not resolve multiple HPV infections determined by LiPA (16.7\% cases). TS-PCR for each of HPV types might be time-consuming, and sequencing inappropriate because most of these types were found as multiple HPV infections. Thus, similar line blot assays like LiPA would be more eligible for the confirmation of HPV typing findings. ${ }^{32}$

A subset of 32 samples was additionally analysed by sequencing, after we could not determine the exact type by comparing RFLP and LiPA results. Three samples, in which HPV 31, 52 and 66 were determined by sequencing showed complete agreement with both, RFLP and LiPA. However, LiPA identified more types in two of those samples, besides HPV 52 and 66 what was the reason for the additional analysis of these samples by sequencing. Partial agreement of sequencing findings with both, RFLP and LiPA results was found in one sample, where sequencing detected HPV 66, while both, RFLP and LiPA detected multiple infections with HPV types 18 and 66. A priori multiple HPV infections could not be resolved by sequencing, thus we can consider this case as an exception to the rule. In cases of multiple HPV infections, RFLP method can be more informative than sequencing, but LiPA is the best choice among those three methods for the simultaneous identification of HPV types.

This study emphasizes the value of different methods of HPV detection and typing. At the moment there is no gold standard for HPV typing. Clinical laboratories, that intend to adopt the HPV typing, should consider the advantages and disadvantages of each of the described method. From the results of this study we can suggest to precede with typing by TS-PCR of the most commonly expected HPV types in the studied population; in Croatia they are LR HPV 6/11 and HR HPV 16, 18, 31, 33 and probably HPV 51, 53, 56, 58 and 66 to be confirmed on a larger study group. Confirmation of single HPV infection can be made by sequencing, if available, rather than RFLP that is cumbersome and time-consuming. The commercially available LiPA (Innogenetics) gives a wide spread of HPV types in one sample, it is suitable for the identification of multiple HPV infection besides single HPV infections and it is easy to perform but it's major limitation is the price of the test. Moreover, the high degree of discordant findings between HPV typing tests in this study indicates that neither test is perfect. Thus, careful consideration should be taken when test are used for clinical purpose.

\section{Acknowledgements}

This work was supported by grant numbers 098-0982464-2510 and 101-0982464-2277 from the Ministry of Science, Technology and Sport of the Republic of Croatia. The authors thank Jasminka Golubić Talić for expert technical assistance. 


\section{Reference}

1. zur Hausen H (2002) Papillomaviruses and cancer: from basic studies to clinical application. Nat Rev Cancer 2:342-350

2. Muñoz N, Castellsagué X, de González AB et al (2006) Chapter 1: HPV in the etiology of human cancer. Vaccine 24:S1-S10

3. Gillison ML, Lowy DR (2004) A causal role for human papillomavirus in head and neck cancer. Lancet 363:1488-1489

4. de Villiers EM, Fauquet C, Broker TR et al (2004) Classification of papillomaviruses. Virology 324:1727

5. Walboomers JM, Jacobs MV, Manos MM et al (1999) Human papillomavirus is a necessary cause of invasive cervical cancer worldwide. J Pathol 189:12-19

6. Muñoz N, Bosch FX, de Sanjosé S et al (2003) Epidemiologic classification of human papillomavirus types associated with cervical cancer. N Engl J Med 348:518-527

7. Muñoz N, Bosch FX, Castellsagué X et al (2004) Against which human papillomavirus types shall we vaccinate and screen? The international perspective. Int J Cancer 111:278-285

8. Grce M, Husnjak K, Bozikov J et al (2001) Evaluation of genital human papillomavirus infections by polymerase chain reaction among Croatian women. Anticancer Res 21:579-584

9. Bosch FX, Lorincz A, Muñoz N et al (2002) The causal relation between human papillomavirus and cervical cancer. J Clin Pathol 55:244-265

10. Iftner T, Villa LL (2003) Chapter 12: Human papillomavirus technologies. J Natl Cancer Inst Monogr $31: 80-88$

11. van den Brule AJ, Meijer CJ, Bakels V et al (1990) Rapid detection of human papillomavirus in cervical scrapes by combined general primer-mediated and type-specific polymerase chain reaction. $\mathbf{J}$ Clin Microbiol 28:2739-2743

12. Soler C, Allibert P, Chardonnet $Y$ et al (1991) Detection of human papillomavirus types 6, 11, 16 and 18 in mucosal and cutaneous lesions by the multiplex polymerase chain reaction. J Virol Methods $35: 143-157$

13. Gravitt PE, Peyton CL, Apple RJ et al (1998) Genotyping of 27 human papillomavirus types by using L1 consensus PCR products by a single-hybridization, reverse line blot detection method. J Clin Microbiol 36:3020-3027

14. Kleter B, van Doorn LJ, Schrauwen L et al (1999) Development and clinical evaluation of a highly sensitive PCR-reverse hybridization line probe assay for detection and identification of anogenital human papillomavirus. J Clin Microbiol 37:2508-2517

15. van den Brule AJ, Pol R, Fransen-Daalmeijer N et al (2000) GP5+/6+ PCR followed by reverse line blot analysis enables rapid and high-throughput identification of human papillomavirus genotypes. $\mathbf{J}$ Clin Microbiol 40:779-787

16. Ovanin-Rakić A, Pajtler M, Stanković T et al (2003) [Classification of cervical cytological smears "Zagreb 2002". Modification of the classification "Zagreb 1990" and "NCI Bethesda 2001".] [In Croatian] Gynaecol Perinatol 12:148-153

17. Milutin-Gašperov N, Sabol I, Halec G et al (2007) Retrospective study of the prevalence of high-risk human papillomaviruses among Croatian women. Coll Antropol, Suppl 2:89-96

18. Maniatis T, Frisch EF, Sambrook J (eds) (1989) Molecular Cloning: A Laboratory Manual. Cold Spring Harbor Laboratory, Cold Spring Harbor, New York

19. Grce M, Husnjak K, Magdić L et al (1997) Detection and typing of human papillomaviruses by polymerase chain reaction in cervical scrapes of Croatian women with abnormal cytology. Eur $\mathrm{J}$ Epidemiol 13:645-651 
20. Husnjak K, Grce M, Magdić L et al (2000) Comparison of five different polymerase chain reaction methods for detection of human papillomavirus in cervical cell specimens. J Virol Methods 88:125-134

21. Bell DA, Taylor JA, Paulson DF et al (1993) Genetic risk and carcinogen exposure: a common inherited defect of the carcinogen-metabolism gene glutathione S-transferase M1 (GSTM1) that increases susceptibility to bladder cancer. J Natl Cancer Inst 85:1159-1164

22. Ting Y, Manos MM (1990) Detection and typing of genital human papillomaviruses. In: Innis MA, Gelfand DH, Sninsky JJ, White TJ (eds) PCR protocols: a guide to methods and applications, Academic Press, San Diego, p 356

23. Meyer T, Arndt R, Stockfleth E et al (1995) Strategy for typing human papillomaviruses by RFLP analysis of PCR products and subsequent hybridization with a generic probe. Biotechniques 19:632-639

24. Bernard HU, Chan SY, Manos MM et al (1994) Identification and assessment of known and novel human papillomaviruses by polymerase chain reaction amplification, restriction fragment length polymorphisms, nucleotide sequence, and phylogenetic algorithms. J Infect Dis 170:1077-1085

25. Quint WG, Scholte G, van Doorn LJ et al (2001) Comparative analysis of human papillomavirus infections in cervical scrapes and biopsy specimens by general SPF(10) PCR and HPV genotyping. J Pathol 194:51-58

26. Altschul SF, Gish W, Miller W et al (1990) Basic local alignment search tool. J Mol Biol 215:403-410

27. Clifford GM, Smith JS, Aguado T et al (2003) Comparison of HPV type distribution in high-grade cervical lesions and cervical cancer: a meta-analysis. Br J Cancer 89:101-105

28. Smith JS, Lindsay L, Hoots B et al (2007) Human papillomavirus type distribution in invasive cervical cancer and high-grade cervical lesions: a meta-analysis update. Int J Cancer 121:621-632

29. Perrons C, Kleter B, Jelley R et al (2002) Detection and genotyping of human papillomavirus DNA by SPF10 and MY09/11 primers in cervical cells taken from women attending a colposcopy clinic. J Med Virol 67:246-252

30. van Doorn LJ, Quint W, Kleter B et al (2002) Genotyping of human papillomavirus in liquid cytology cervical specimens by the PGMY line blot assay and the $\operatorname{SPF}(10)$ line probe assay. J Clin Microbiol 40:979-983

31. Gillio-Tos A, De Marco L, Ghisetti V et al (2006) Human papillomavirus typing with GP5+/6+ polymerase chain reaction reverse line blotting and with commercial type-specific PCR kits. J Clin Virol 36:126-132

32. Sabol I, Salakova M, Smahelova J et al (2008) Evaluation of different techniques for the identification of human papillomavirus types of low prevalence. J Clin Microbiol (in press). DOI 10.1128/JCM.02328-07 


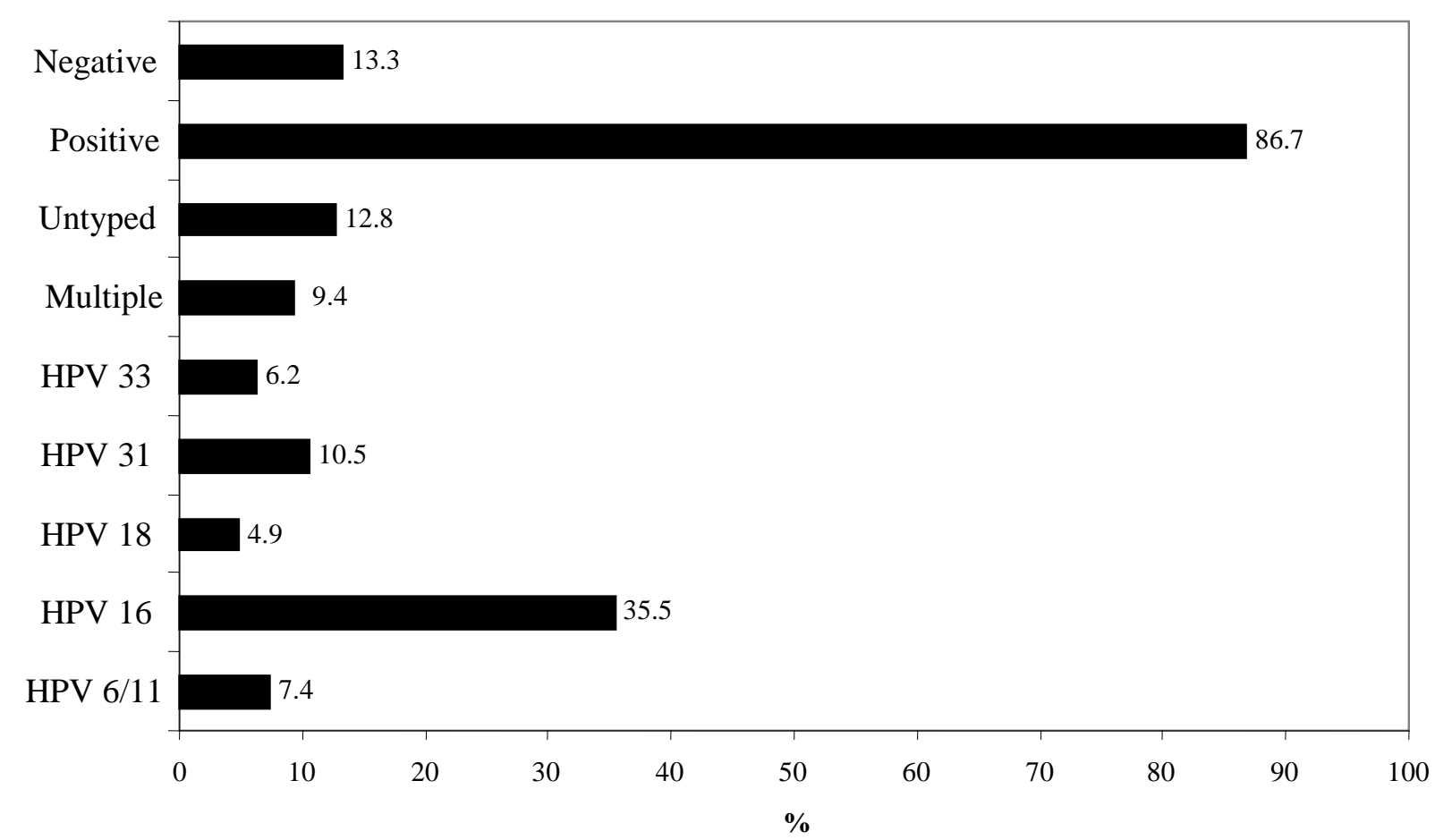

Figure 1. Prevalence of common human papillomavirus (HPV) types in high-grade squamous intraepithelial lesions (HSIL) samples obtained by polymerase chain reaction (PCR) with consensus primers (MY09/MY11, L1C1/L1C2-1/L1C2-2 and/or GP5/6) and type-specific primers (TS) for HPV 6/11, 16, 18, 31 and 33 
Table 1. HPV genotyping by RFLP, LiPA and sequencing

\begin{tabular}{|c|c|c|c|}
\hline $\begin{array}{c}\text { Number of } \\
\text { cases }\end{array}$ & $\begin{array}{c}R F L P \\
(N=88)\end{array}$ & $\begin{array}{c}L i P A \\
(N=88)\end{array}$ & $\begin{array}{c}\text { Sequencing } \\
\quad(N=32)\end{array}$ \\
\hline 2 & 16 & 16 & 1 \\
\hline 1 & 31 & 31 & I \\
\hline 1 & 31 & 31 & 31 \\
\hline 1 & 33 & 53 & 33 \\
\hline 1 & 33 & 33 & 1 \\
\hline 1 & 33 & ND & 33 \\
\hline 1 & 34 & $34 / 51 / 52$ & 1 \\
\hline 1 & 39 & $39 / 51$ & 1 \\
\hline 1 & 45 or 56 & $45 / 52$ & 45 \\
\hline 1 & ND & 45 & 45 \\
\hline 2 & ND & 52 & 52 \\
\hline 1 & 52 & $52 / 68$ or 73 & 52 \\
\hline 1 & 52 & 52 & 1 \\
\hline 10 & 53 & 53 & I \\
\hline 2 & 53 & $52 / 53$ & I \\
\hline 5 & 56 & 56 & I \\
\hline 1 & ND & ND & 56 \\
\hline 7 & 58 & 58 & 1 \\
\hline 1 & ND & 58 & 58 \\
\hline 1 & 58 & $52 / 58$ & 1 \\
\hline 3 & 59 & 59 & / \\
\hline 1 & 61 & 51 & 61 \\
\hline 1 & 61 & 52 & 61 \\
\hline 1 & 61 & 53 & 61 \\
\hline 1 & 26 or 62 & 51 & 62 \\
\hline 3 & ND & 66 & 66 \\
\hline 1 & 66 & $52 / 66$ & 66 \\
\hline 2 & 66 & 66 & / \\
\hline 1 & 66 & $66 / 70^{*}$ & I \\
\hline 1 & 67 & ND & 67 \\
\hline 2 & 68 & 68 or 73 & 1 \\
\hline 1 & ND & $51 / 70$ & 70 \\
\hline 1 & ND & 70 & 70 \\
\hline 1 & 73 & 68 or 73 & 73 \\
\hline 1 & 26 or 62 & 52 & 81 \\
\hline 1 & 83 & ND & 83 \\
\hline 1 & 84 & 42 & 84 \\
\hline 1 & ND & 66 & 84 \\
\hline 1 & 64 or 84 & 66 & 84 \\
\hline 1 & $18 / 66$ & $18 / 66$ & 66 \\
\hline 1 & ND & $52 / 56 / 66$ & ND \\
\hline 1 & ND & 45 & 82 \\
\hline 1 & ND & 45 & I \\
\hline 1 & $33 / 59$ & $33 / 59 / 39 / 52$ & I \\
\hline 1 & 58 & $58 / 51 / 68$ or 73 & I \\
\hline 1 & $55 / 58$ & ND & I \\
\hline 1 & Multiple & $39 / 51 / 70$ & I \\
\hline 1 & $52 / 61$ & $39 / 52$ & I \\
\hline 1 & Multiple & $66 / 68$ or 73 & 1 \\
\hline 1 & $53 / 73$ & $53 / 68$ & Multiple \\
\hline 1 & Multiple & $51 / 53 / 68$ or 73 & 1 \\
\hline 1 & Multiple & ND & I \\
\hline 1 & Multiple & ND & Multiple \\
\hline 1 & Multiple & $51 / 52 / 66$ & 1 \\
\hline 1 & Multiple & $35 / 53 / 66$ & I \\
\hline 1 & Multiple & $31 / 40 / 58 / 53$ & I \\
\hline 1 & ND & $39 / 51 / 66$ & I \\
\hline 1 & 73 & $39 / 68$ or 73 & IS223 \\
\hline 1 & ND & 70 & SDL105 \\
\hline 1 & 73 & 68 or 73 & IS223 \\
\hline
\end{tabular}

ND - not determined; *HPV 70, very weak signal 


\section{Table 2. Detection of HPV infections by RFLP and LiPA assays}

\begin{tabular}{lcc}
\hline $\begin{array}{l}\text { Type of } H P V \\
\text { infection }(N=88)\end{array}$ & $\begin{array}{c}\text { RFLP } \\
\text { No. }(\%)\end{array}$ & $\begin{array}{c}\text { LiPA } \\
\text { No. }(\%)\end{array}$ \\
\hline Single & $59(67.0)$ & $57(64.8)$ \\
Multiple & $13(14.8)$ & $24(27.3)$ \\
Undetermined & $16(18.2)$ & $7(8.0)$ \\
\hline
\end{tabular}

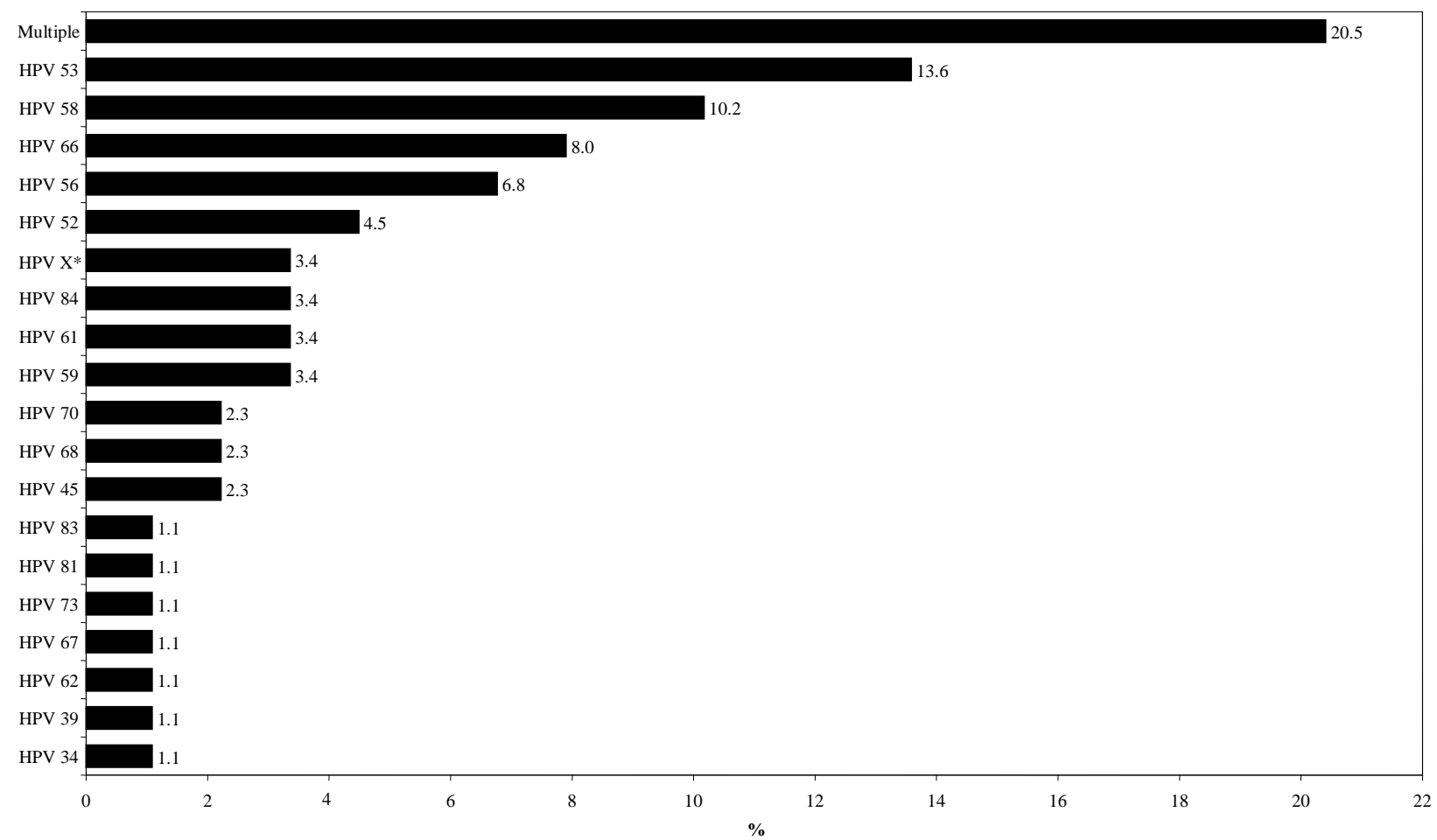

Figure 2. Frequencies of low abundant human papillomavirus $(H P V)$ types in the subset $(N=88)$ of highgrade squamous intraepithelial lesions (HSIL) samples positive by consensus PCR and negative by TS-PCR for $6 / 11,16,18,31$ and 33, obtained by combining results of restriction fragment length polymorphism (RFLP) analysis, line probe assay (LiPA, Innogenetics) and sequencing; *incompletely determined HPV type 
Table 3. Identification of a particular HPV type either by RFLP or LiPA and agreement between these two assays given as Kappa values

\begin{tabular}{lcccc}
\hline HPV type & RFLP & LiPA & Kappa & $95 \%$ CI \\
\hline 16 & 2 & 2 & 1.000 & 1.000 to 1.000 \\
18 & 1 & 1 & 1.000 & 1.000 to 1.000 \\
$26^{*}$ & 2 & 0 & 0.000 & -1.370 to 1.370 \\
31 & 2 & 3 & 0.794 & 0.394 to 1.195 \\
33 & 4 & 2 & 0.656 & 0.185 to 1.127 \\
34 & 1 & 1 & 1.000 & 1.000 to 1.000 \\
35 & 0 & 1 & 0.000 & -1.949 to 1.949 \\
39 & 1 & 6 & 0.272 & -0.349 to 0.892 \\
40 & 0 & 1 & 0.000 & -1.949 to 1.949 \\
42 & 0 & 1 & 0.000 & -1.949 to 1.949 \\
45 & 1 & 4 & 0.389 & -0.291 to 1.069 \\
51 & 0 & 10 & 0.000 & -0.584 to 0.584 \\
52 & 3 & 16 & 0.274 & -0.090 to 0.638 \\
53 & 13 & 18 & 0.805 & 0.640 to 0.971 \\
$55^{*}$ & 1 & 0 & 0.000 & -1.949 to 1.949 \\
56 & 6 & 6 & 0.821 & 0.576 to 1.066 \\
58 & 10 & 11 & 0.838 & 0.657 to 1.018 \\
59 & 4 & 4 & 1.000 & 1.000 to 1.000 \\
$61^{*}$ & 4 & 0 & 0.000 & -0.957 to 0.957 \\
$62^{*}$ & 2 & 0 & 0.000 & -1.370 to 1.370 \\
$64^{*}$ & 1 & 0 & 0.000 & -1.949 to 1.949 \\
66 & 5 & 15 & 0.453 & 0.134 to 0.772 \\
$67^{*}$ & 1 & 0 & 0.000 & -1.949 to 1.949 \\
68 & 2 & 10 & 0.307 & -0.151 to 0.765 \\
70 & 0 & 5 & 0.000 & -0.851 to 0.851 \\
73 & 4 & 10 & 0.542 & 0.188 to 0.896 \\
$83^{*}$ & 1 & 0 & 0.000 & -1.949 to 1.949 \\
$84^{*}$ & 2 & 0 & 0.000 & -1.370 to 1.370 \\
\hline & & & &
\end{tabular}

*HPV types that LiPA cannot detect

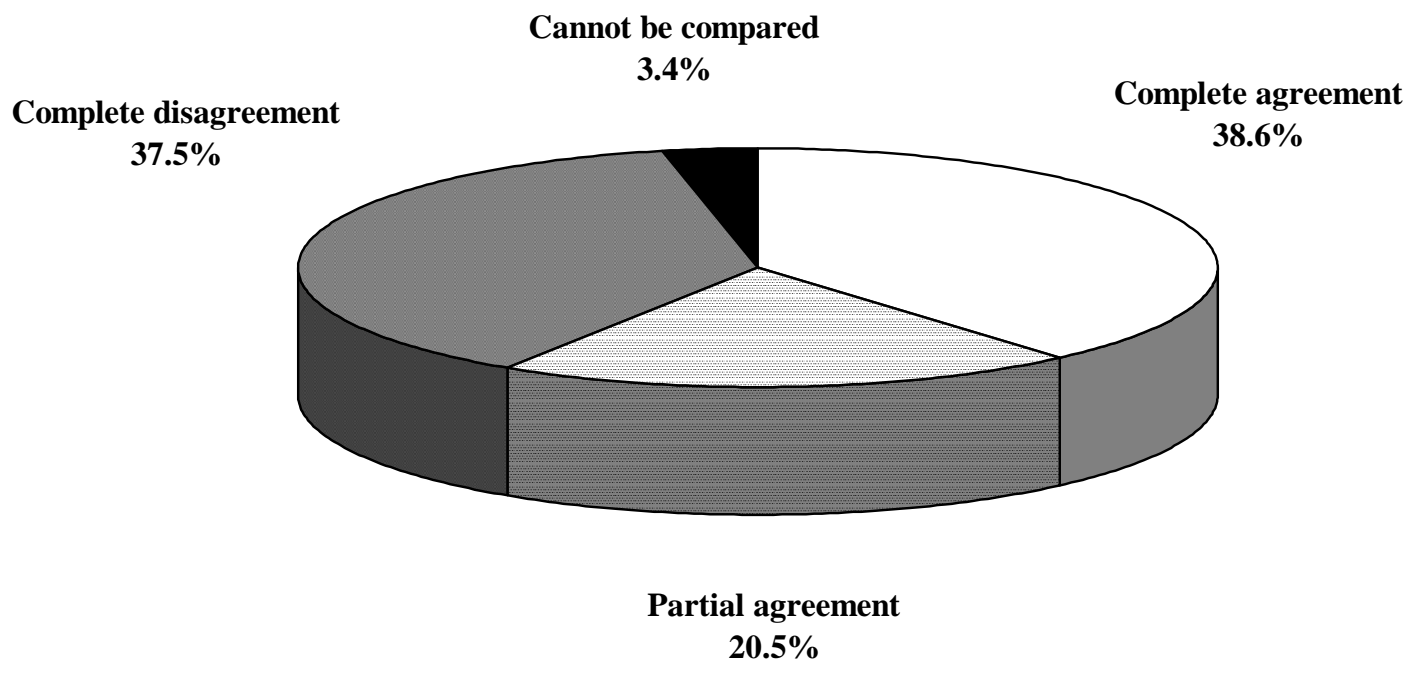

Figure 3. Comparison of HPV typing by RFLP and LiPA $(N=88)$ 